\title{
Quantum Breaking Bound on de Sitter and Swampland
}

\author{
Gia Dvali ${ }^{a, b, c}$, Cesar Gomez ${ }^{d}$ and Sebastian Zell ${ }^{a, b}$ \\ ${ }^{a}$ Arnold Sommerfeld Center, Ludwig-Maximilians-Universität, Theresienstraße 37, 80333 München, Germany \\ ${ }^{b}$ Max-Planck-Institut für Physik, Föhringer Ring 6, 80805 München, Germany \\ ${ }^{c}$ Center for Cosmology and Particle Physics, Department of Physics, \\ New York University, 726 Broadway, New York, NY 10003, USA and \\ ${ }^{d}$ Instituto de Física Teórica UAM-CSIC, Universidad Autónoma de Madrid, Cantoblanco, 28049 Madrid, Spain
}

\begin{abstract}
Quantum consistency suggests that any de Sitter patch that lasts a number of Hubble times that exceeds its Gibbons-Hawking entropy divided by the number of light particle species suffers an effect of quantum breaking. Inclusion of other interactions makes the quantum break-time shorter. The requirement that this must not happen puts severe constraints on scalar potentials, essentially suppressing the self-reproduction regimes. In particular, it eliminates both local and global minima with positive energy densities and imposes a general upper bound on the number of e-foldings in any given Hubble patch. Consequently, maxima and other tachyonic directions must be curved stronger than the corresponding Hubble parameter. We show that the key relations of the recently-proposed de Sitter swampland conjecture follow from the de Sitter quantum breaking bound. We give a general derivation and also illustrate this on a concrete example of $D$-brane inflation. We can say that string theory as a consistent theory of quantum gravity nullifies a positive vacuum energy in self-defense against quantum breaking.
\end{abstract}

As the quantum picture shows, any de Sitter patch with Hubble parameter $H$ undergoes a phenomenon of quantum breaking after the time [1-3]

$$
t_{Q}=\frac{1}{N_{s p}} \frac{M_{P}^{2}}{H^{3}}
$$

where $N_{s p}$ is the number of light particle species [3]. Since $S_{G H} \equiv M_{P}^{2} / H^{2}$ is an effective Gibbons-Hawking entropy of de Sitter, the relation (11) closely resembles the half-life time of a black hole in a theory with $N_{s p}$ particle species. This resemblance is no accident. As it is known [4], black holes impose an absolute non-perturbative upper bound of $M_{P} / \sqrt{N_{s p}}$ on the cutoff scale of quantum gravity.

While a macroscopic black hole decays away via Hawking evaporation, the story with de Sitter is more problematic. A system stuck in a de Sitter phase because of a positive vacuum energy is bound to face quantum breaking unless there exists a degree of freedom (e.g., an inflaton) that ends the de Sitter phase prior to its quantum breakdown in every Hubble patch.

It is important to understand that the quantum breaking phenomenon of de Sitter identified in [1-3] is a fully microscopic non-perturbative collective phenomenon unrelated to any breakdown of naive perturbation theory. It marks the point beyond which the true quantum evolution no longer matches any semi-classical counterpart. As such it represents a consistency challenge rather than an artifact of a wrong formalism. Nevertheless, glimpses of an analogous time scale can be read off as semi-classical IR-effects [5]. Thus, quantum breaking provides a possible microscopic meaning to these effects.

The requirement that quantum breaking should not happen implies that each Hubble patch must exit the de Sitter state beforehand, i.e., it restricts from above the number of e-foldings that any given Hubble patch is allowed to experience prior to exiting the de Sitter phase by [6]

$$
\mathcal{N}_{\max }=\frac{1}{N_{s p}} \frac{M_{P}^{2}}{H^{2}}
$$

This puts severe restrictions on scalar potentials. Essentially, it excludes any potential that can allow the regime of self-reproduction [9] since, as it is well known, in such cases in some Hubble patches the de Sitter phase can last eternally and certainly longer than $t_{Q}$. This excludes potentials with local or global minima with positive energy densities as well as any eternally inflating section of the scalar potential. An immediate implication of the bound (2) is that today's dark energy cannot be constant [1 3$]$.

In order to avoid a confusion, we must stress that the bound (2) per se does not exclude the possibility of some exotic future-eternal state past the quantum break-time. What is evident is that the mean field description of such a quantum state shall no longer match any reasonable classical metric solution of GR. Such a scenario was termed as quantum eternity in [1]. While currently we cannot exclude this interesting possibility from first principles, we see no supporting evidence for it. In this note therefore we take (2) as a consistency bound.

As it was shown in [3], for a system with generic interactions the quantum break-time is given by

$$
t_{Q}=\frac{t_{C l}}{\alpha}
$$

where $t_{C l}$ is a characteristic classical time scale of the system while $\alpha$ is the strength of the relevant interaction that leads to quantum breaking. Then the relation (1) represents a particular form of (3) applied to de Sitter with pure gravity. In this case $t_{C l}=H^{-1}$ is the Hubble time and $\alpha=N_{s p} H^{2} / M_{P}^{2}$ is an effective strength 
of graviton scattering for the characteristic momentum transfer $H$. The general quantum breaking bound tells us that the state of the system must evolve significantly on a time scale $t_{e s c}$ that is shorter than $t_{Q}$ :

$$
t_{e s c} \lesssim t_{Q}
$$

As said, this condition puts severe consistency restrictions on the form of scalar potentials.

Recently [10], a swampland de Sitter conjecture has been proposed, which imposes analogous constraints but from a different consideration (some cosmological implications were discussed shortly after in [11]). In a previous note [12], we have pointed to some close similarities between the new proposal [10] and the de Sitter quantum breaking bound of [1-3].

The purpose of this short note is to deepen this connection with [10] including its more recent refined version [13]. The touching point is that both proposals exclude self-reproduction regimes and this results in natural similarities in the constraints that they impose on scalar potentials. We shall illustrate the connection for the key regimes by highlighting similarities of the bounds imposed by the two proposals on unstable extrema and on slow-roll potentials. Some recent discussions of the swampland conjecture can be found in [14-25].

Bound on Extrema. We shall restrict ourselves to positive-semidefinite potentials. Consider such a scalar field potential $V(\phi)$ in a neighborhood of an unstable extremum. This may be either a local maximum or a tachyonic direction around a saddle point with a negative curvature $V^{\prime \prime}$.

Now if the absolute value of this curvature $\left|V^{\prime \prime}\right|$ is much larger than the Hubble parameter $H^{2}=V / M_{P}^{2}$, the field is unstable and leaves the neighborhood on a time scale $t_{e s c} \sim\left|V^{\prime \prime}\right|^{-\frac{1}{2}}$, which is much shorter than the Hubble time $H^{-1}$. Therefore, the system has no chance to suffer from de Sitter quantum breaking.

In the opposite case $\left|V^{\prime \prime}\right| \ll H^{2}$, the field behaves as effectively-massless. It then experiences a random walk with variation $\delta \phi \sim H$ per Hubble volume per Hubble time. These quantum excursions lead to a selfreproduction of the de Sitter phase [9]. Namely, there always exists a Hubble patch in which the field would stay on top of the hill longer than the quantum break-time (1). Thus, such a regime violates the quantum breaking constraint (44). Avoidance of this violation implies the following bound

$$
V^{\prime \prime} \lesssim-V / M_{P}^{2}
$$

which is exactly the one proposed in [14], [15], [13]. Thus, quantum breaking exposes the fundamental meaning of this expression from basic principles of the quantum theory.

Bound on Slow-Roll. We now move to another example and show how quantum breaking constrains the slow-roll regime. We thus consider the potential $V(\phi)$ away from extrema. The quantum breaking bound (4) demands that the change of the potential $\Delta V$ over some time $\Delta t \sim t_{Q}$ must satisfy $|\Delta V| \gtrsim V$. Approximating $\Delta V \sim V^{\prime} \dot{\phi} \Delta t$ and assuming that slow roll is satisfied, $\dot{\phi} \sim-V^{\prime} / H$, we get $\Delta V \sim-V^{\prime 2} \Delta t / H$. Using (3) and taking into account that in the slow-roll case $t_{C l} \lesssim H^{-1}$, we arrive at the following bound:

$$
\frac{M_{P}\left|V^{\prime}\right|}{V} \gtrsim \sqrt{\alpha}
$$

Notice that this bound is stronger than the one presented in [12] by a factor $1 / \sqrt{\alpha}$. Both bounds have the form of the one conjectured in [10], depending on whether we identify the coefficient as $c=\sqrt{\alpha}$ or $c=\alpha$. Also as already pointed out in 12], in our case $\alpha$ is not necessarily a fixed constant but can depend on $\phi$ and $V$, for example as it is the case in pure gravity.

But the important point is that the fundamental physical meaning is made transparent. Namely, the slow-roll parameter is bounded from below by the strength of the quantum coupling. If the coupling is stronger, the quantum break-time becomes shorter and the system needs to move faster to avoid it!

Note that the quantum breaking bound does not exclude inflation but restricts the number of e-foldings in any given Hubble patch by:

$$
\mathcal{N}_{\text {max }} \sim \alpha^{-1} .
$$

This gives (2) for purely gravitational coupling but is more general.

The quantum breaking bound leaves enough room for inflationary model building. For example, scenarios such as topological inflation [26] or hilltop inflation [27], in which inflation occurs near maxima, are not necessarily incompatible with the quantum breaking bound but require closer scrutiny in order to limit the number of e-foldings by (2). Furthermore, we remark that this bound looks pretty mild since for small values of $\alpha$ the number $\mathcal{N}_{\text {max }}$ is large. However, when translated into constraints on the scalar potential, we realize that the bounds (2) and (7) are sufficiently stringent, due to an exponential sensitivity of $\mathcal{N}_{\max }$ to the curvature of $V$.

Constraints in String Theory. Moving to string theoretic inflationary model building, we are fully aware that within specific compactification frameworks constraints may become much more severe, as this is expressed in [10]. This was apparent already since the early models of inflation driven by $D$-branes [28]. From our perspective, the difficulties in generating a high number of e-foldings in such setups can be viewed as the fast escape of the system from a would-be de Sitter phase in avoidance of disastrous quantum breaking.

Let us illustrate the quantum breaking bound at work on a simple example of a stringy realization of an un- 
stable extremum. It is given by a $D$-brane system that was originally proposed as a framework for stringy inflation [28]. For definiteness, we choose a system of a $D_{3^{-}}$and an anti- $D_{3}$-brane in a 10 -dimensional space on which 6 extra dimensions have been compactified with characteristic radii $R$ much larger than the string length $L_{s}$. The precise topology is not important for the current purposes since we shall consider the $D_{3}-\bar{D}_{3}$ to be aligned with 4 non-compact dimensions and separated in the external space by a distance that is much shorter than the compactification radius $R$. In this case, the branes fall towards each other due to the force mediated by a tree-level closed string exchange. In [28] this process was mapped on an inflationary slow-roll.

Once the branes approach each other at a distance of order of the string length and start to overlap, an open string mode becomes tachyonic with the mass ${ }^{2}$ given by $-m_{s}^{2}$, where $m_{s}=L_{s}^{-1}$ is the string scale. Although a complete form of the potential for the tachyon is not known, this is not important for our estimates since we are only interested in the curvature around the maximum which is set by the tachyon mass, $V^{\prime \prime}=-m_{s}^{2}$. This sets the escape time, during which the system relaxes, roughly as $t_{e s c} \sim L_{s} \ln \left(g_{s}^{-1}\right)$. This relaxation time must be compared to the quantum break-time which is given by $t_{Q} \sim \frac{L_{s}}{g_{s}^{2}}$. If the string theory is weakly-coupled, the latter time scale is obviously longer than the time $t_{e s c}$ that the system needs to relax from the top of the hill. Thus, the quantum breaking bound (4) is satisfied.

We can now understand this result in the language of the relation (5). Indeed we have

$$
H^{2} \sim \frac{m_{s}^{4}}{g_{s} M_{P}^{2}}=m_{s}^{2} \frac{g_{s}}{\left(m_{s} R\right)^{6}}=-V^{\prime \prime} \frac{g_{s}}{\left(R / L_{s}\right)^{6}},
$$

where we have used the well-known relations between the 10-dimensional Planck and string scales $\frac{m_{s}^{8}}{g_{s}^{2}}=M_{10}^{8}$ and the 10- and 4-dimensional Planck masses $M_{P}^{2}=M_{10}^{8} R^{6}$. Since $g_{s}$ is weak and $R \gg L_{s}$, we immediately conclude that the 10-dimensional stringy quantum breaking bound translates into the 4-dimensional relation (5).

In summary, an apparent "de Sitter-phobia" of string theory can be interpreted as a manifestation of the fact that as a consistent theory of quantum gravity, string theory puts up a "self-defense" mechanism against the de Sitter quantum breaking. In this respect we could say that via the bound (2), quantum gravity nullifies the cosmological constant problem by promoting it from a naturalness problem into a problem of quantum consistency [2]. As it was already predicted in [1], an obvious consequence of the quantum breaking bound is that the currently observed dark energy cannot be constant and must change in time. Our lower bound, however, gives enough room for a variation not to be easily detectable by observations. Therefore, it is important to cross-check the idea by looking for imprints of quantum breaking in the spectrum of primordial inflationary perturbations. Some leading corrections were estimated in [1].

Acknowledgements. This work was supported in part by the Humboldt Foundation under Humboldt Professorship Award and ERC Advanced Grant 339169 "Selfcompletion".
[1] G. Dvali, C. Gomez, Quantum Compositeness of Gravity: Black Holes, AdS and Inflation, JCAP 1401 (2014) 023, arXiv:1312.4795 [hep-th].

[2] G. Dvali , C. Gomez, Quantum Exclusion of Positive Cosmological Constant?, Annalen Phys. 528 (2016) 68-73, arXiv:1412.8077 [hep-th].

[3] G. Dvali, C. Gomez, S. Zell, Quantum Break-Time of de Sitter, JCAP 1706 (2017) 028, arXiv:1701.08776 [hep-th].

[4] G. Dvali, Black Holes and Large N Species Solution to the Hierarchy Problem, Fortsch. Phys. 58 (2010) 528, arXiv:0706.2050 [hep-th];

G. Dvali, M. Redi, Black Hole Bound on the Number of Species and Quantum Gravity at LHC, Phys. Rev. D77 (2008) 045027, arXiv:0710.4344 [hep-th];

G. Dvali, C. Gomez, Quantum Information and Gravity Cutoff in Theories with Species, Phys. Lett. B674 (2009) 303-307, arXiv:0812.1940 [hep-th].

[5] U. H. Danielsson, D. Domert, and M. E. Olsson, Puzzles and resolutions of information duplication in de Sitter space, Phys. Rev. D 68 (2003) 083508, arXiv:hepth/0210198;

S. B. Giddings and M. S. Sloth, Semiclassical relations and IR effects in de Sitter and slow-roll space-times,
JCAP 1101 (2011) 023, arXiv:1005.1056 [hep-th];

R. Z. Ferreira, M. Sandora, and M. S. Sloth, Asymptotic Symmetries in de Sitter and Inflationary Spacetimes, JCAP 1704 (2017) 033, arXiv:1609.06318 [hep-th]; Patient Observers and Non-perturbative Infrared Dynamics in Inflation, JCAP 1802 (2018) 055, arXiv:1703.10162 [hep-th];

T. Markkanen, De Sitter Stability and Coarse Graining, Eur.Phys.J. C78 (2018) 97, arXiv:1703.06898 [gr-qc].

[6] In [1, 3] this bound was derived from quantum breaking due to a collective effect of purely graviton scattering. The relation (2) is therefore model-independent. In [1] it was also suggested that analogous inflaton-graviton scattering could lead to a second bound $\mathcal{N}_{\text {max }}=\left(M_{P} / H\right)^{\frac{4}{3}}$. However, here we adopt the model-independent gravitational bound (2). For more discussions within this framework, see [7]. A similar restriction (without considering species effect) on the number of e-foldings from holographic considerations was suggested earlier in [8].

[7] F. Kuhnel, M. Sandstad, Corpuscular Consideration of Eternal Inflation, Eur. Phys.J. C75 (2015) 505, arXiv:1504.02377 [gr-qc];

L. Berezhiani, On Corpuscular Theory of Inflation, Eur. 
Phys. J. C77 (2017) 106, arXiv:1610.08433 [hep-th]; R. Casadio, A. Giugno, A. Giusti, Corpuscular slow-roll inflation, Phys. Rev. D97 (2018) 024041, arXiv:1708.09736 [gr-qc].

[8] N. Arkani-Hamed, S. Dubovsky, A. Nicolis, E. Trincherini, and G. Villadoro, A Measure of de Sitter entropy and eternal inflation, JHEP 05 (2007) 055, arXiv:0704.1814 [hep-th].

[9] A. D. Linde, Eternally Existing Self-reproducing Chaotic Inflationary Universe, Phys. Lett. B 175 (1986) 395.

A. H. Guth, Eternal inflation and its implications, J. Phys. A40 (2007) 6811, arXiv:hep-th/0702178.

[10] G. Obied, H. Ooguri, L. Spodyneiko, C. Vafa, De Sitter Space and the Swampland, arXiv:1806.08362 [hep-th].

[11] P. Agrawal, G. Obied, P. J. Steinhardt, C. Vafa, On the Cosmological Implications of the String Swampland, Phys.Lett. B784 (2018) 271-276 arXiv:1806.09718 [hepth].

[12] G. Dvali, C. Gomez, On Exclusion of Positive Cosmological Constant, arXiv:1806.10877 [hep-th].

[13] H. Ooguri, E. Palti, G. Shiu, C. Vafa, Distance and de Sitter Conjectures on the Swampland, arXiv:1810.05506 [hep-th].

[14] D. Andriot, On the de Sitter swampland criterion, arXiv:1806.10999 [hep-th].

[15] S. K. Garg, C. Krishnan, Bounds on Slow Roll and the de Sitter Swampland, arXiv:1807.05193 [hep-th].

[16] A. Kehagias and A. Riotto, A note on Inflation and the Swampland, arXiv:1807.05445 [hep-th].

[17] F. Denef, A. Hebecker, T. Wrase, de Sitter swampland conjecture and the Higgs potential, Phys.Rev. D98 (2018) 086004, arXiv:1807.06581 [hep-th].
[18] L. Heisenberg, M. Bartelmann, R. Brandenberger, and A. Refregier, Dark Energy in the Swampland, Phys. Rev. D 9 (2018) 123502, arXiv:1808.02877 [astro-ph.CO].

[19] W. H. Kinney, S. Vagnozzi and L. Visinelli, The Zoo Plot Meets the Swampland: Mutual (In)Consistency of Single-Field Inflation, String Conjectures, and Cosmological Data, arXiv:1808.06424 [astro-ph.CO].

[20] K. Choi, D. Chway and C. S. Shin, The dS swampland conjecture with the electroweak symmetry and QCD chiral symmetry breaking, arXiv:1809.01475 [hep-th].

[21] U. Danielsson, The quantum swampland, arXiv:1809.04512 [hep-th].

[22] M. Motaharfar, V. Kamali and R. O. Ramos, Warm way out of the Swampland, arXiv:1810.02816 [astro-ph.CO].

[23] A. Ashoorioon, Rescuing single field inflation from the swampland, Phys. Lett. B 790 (2019) 568, arXiv:1810.04001 [hep-th].

[24] H. Fukuda, R. Saito, S. Shirai and M. Yamazaki, Phenomenological Consequences of the Refined Swampland Conjecture, arXiv:1810.06532 [hep-th].

[25] A. Hebecker and T. Wrase, The asymptotic dS Swampland Conjecture - a simplified derivation and a potential loophole, arXiv:1810.08182 [hep-th].

[26] A. Vilenkin, Topological inflation, Phys.Rev.Lett. 72 (1994) 3137-3140, arXiv:hep-th/9402085;

A. D. Linde, Monopoles as big as a universe, Phys. Lett. B327 (1994) 208, arXiv:astro-ph/9402031.

[27] L. Boubekeur, D. H. Lyth, Hilltop inflation, JCAP 0507 (2005) 010, arXiv:hep-ph/0502047.

[28] G. Dvali, S. H. H. Tye, Brane inflation, Phys. Lett. B450 (1999) 72, arXiv:hep-ph/9812483;

G. Dvali, Q. Shafi, S. Solganik, D-brane inflation, arXiv:hep-th/0105203. 